\title{
Effects and molecular mechanism of pachymic acid on ferroptosis in renal ischemia reperfusion injury
}

\author{
GUI-PING JIANG, YUE-JUAN LIAO, LI-LI HUANG, XU-JIA ZENG and XIAO-HUI LIAO
}

Department of Nephrology, The Second Affiliated Hospital, Chongqing Medical University, Chongqing 400000, P.R. China

Received June 30, 2020; Accepted October 27, 2020

DOI: $10.3892 / \mathrm{mmr} .2020 .11704$

\begin{abstract}
Acute kidney injury (AKI) is a common clinical disease. Ferropotosis, a new type of regulatory cell death, serves an important regulatory role in AKI. Pachymic acid (PA), a lanostane-type triterpenoid from Poria cocos, has been reported to be protective against AKI. However, the protective mechanism of PA in AKI is not yet fully understood. The present study aimed to investigate the effect and molecular mechanism of PA on ferroptosis in renal ischemia reperfusion injury in vivo. A total of 30 mice were intraperitoneally injected with 5,10 and $20 \mathrm{mg} / \mathrm{kg}$ PA for 3 days. A bilateral renal pedicle clip was used for $40 \mathrm{~min}$ to induce renal ischemia-reperfusion injury and establish the model. The results demonstrated that treatment with PA decreased serum creatinine and blood urea nitrogen, and ameliorated renal pathological damage. Transmission electron microscopy revealed no characteristic changes in ferroptosis in the mitochondria of the renal tissue in the high-dose PA group, and only mild edema. Furthermore, treatment with PA increased glutathione expression, and decreased the expression levels of malondialdehyde and cyclooxygenase 2 . Treatment with PA enhanced the protein and mRNA expression levels of the ferroptosis related proteins, glutathione peroxidase 4 (GPX4), solute carrier family 7 (cationic amino acid transporter, $\mathrm{y}+$ system) member 11 (SLC7A11) and heme oxygenase 1 (HO-1) in the kidney, and increased the expression levels of nuclear factor erythroid derived 2 like 2 (NRF2) signaling pathway
\end{abstract}

Correspondence to: Professor Xiao-Hui Liao, Department of Nephrology, The Second Affiliated Hospital, Chongqing Medical University, 76 Linjiang Road, Chongqing 400000, P.R. China

E-mail: 1xh@hospital.cqmu.edu.cn

Abbreviations: AKI, acute kidney injury; BUN, blood urea nitrogen; COX-2, cyclooxygenase 2; GPX4, glutathione peroxidase 4; GSH, glutathione; H\&E, hematoxylin and eosin; HO-1, heme oxygenase 1; IRI, ischemia reperfusion injury; MDA, malondialdehyde; NRF2, nuclear factor erythroid derived 2 like 2; PA, pachymic acid; Scr, serum creatinine; SLC7A11, solute carrier family 7 (cationic amino acid transporter, y+ system) member 11

Key words: pachymic acid, acute kidney injury, renal ischemia reperfusion injury, ferroptosis members. Taken together, the results of the present study suggest that PA has a protective effect on ischemia-reperfusion induced acute kidney injury in mice, which may be associated with the inhibition of ferroptosis in the kidneys through direct or indirect activation of NRF2, and upregulation of the expression of the downstream ferroptosis related proteins, GPX4, SLC7A11 and HO-1.

\section{Introduction}

Acute kidney injury (AKI) is a common clinical disease that is caused by multiple etiologies and has a complex pathophysiological process (1). The morbidity and mortality rates for AKI are high among adults and children, thus, resulting in substantial hospitalization costs $(2,3)$. A meta-analysis of global incidence of AKI revealed incidence rates of AKI were $21.6 \%$ in adults and $33.7 \%$ in children and AKI-associated mortality rates were $23.9 \%$ in adults and $13.8 \%$ in children (4). AKI occurs in $20 \%$ of hospitalized patients, $10 \%$ of whom require renal replacement therapy, and in as much as $50 \%$ of patients in intensive care units, thus significantly increasing the length and cost of hospitalization (5). AKI is characterized by a sharp decrease in glomerular filtration rate in the short term, which is accompanied by an increase in serum creatinine, oliguria or both; these changes can lead to chronic kidney disease or end-stage renal disease (6). Due to the variability of renal injury, the lack of reliable early biomarkers and the heterogeneity of pathways involved in pathophysiology, there are currently no effective means of prevention and intervention except conservative treatment and renal replacement therapy (7). Thus, identifying novel treatment regimens and drugs for AKI is particularly important. The past decade has seen a wealth of research expanding the traditional view of necrosis. Recent research has challenged traditional views of cell death by identifying novel pathways in which cells die in a regulated manner, but exhibit the morphologic features of necrosis (7-9). This regulated necrosis takes several forms: Necroptosis, ferroptosis, pyroptosis, mitochondrial permeability transition-driven necrosis and parthanatos (10). Necroptosis and ferroptosis are the most studied forms of regulated necrosis occurring in the kidney (11). A previous study demonstrated that inhibiting ferroptosis effectively ameliorates AKI (12).

Ferropotosis is a new type of regulatory cell death, which was first proposed by Dixon et al in 2012 (13). This 
type of cell death is iron-dependent and is accompanied by massive iron accumulation and lipid peroxidation during cell death (13). Ferroptosis serves an important regulatory role in the occurrence and development of several diseases, including tumors, neurological diseases and AKI (14). In an in vivo study, ferrostatin 1 or 16-86 was administered 15 min before ischemia in mice with severe ischemia reperfusion injury (IRI)-AKI, and renal tissue damage, serum creatinine and urea all decreased in the mice $48 \mathrm{~h}$ after ischemia, thus, suggesting that ferroptosis has a crucial role in the pathogenesis of IRI (15). Therefore, regulating iron homeostasis, resisting lipid peroxidation and inhibiting ferroptosis may provide a promising therapeutic strategy for AKI.

Pachymic acid (PA), a lanostane-type triterpenoid from Poria cocos, has several pharmacological effects, such as antitumor, anti-inflammatory, antioxidative, hypoglycemic, sedative and hypnotic activities (16-20). A previous study has reported that PA can improve renal injury during sepsis in rats by inhibiting inflammatory responses and activating the nuclear factor erythroid derived 2 like 2 (NRF2)/heme oxygenase 1 (HO-1) pathway through antioxidant stress (21). In addition, Poricoic acid A effectively enhances the inhibition of melatonin in the transition from AKI to chronic kidney disease following renal ischemia/reperfusion injury (22). PA protects against AKI, however, this protective mechanism remains to be further investigated. PA has been reported to activate NRF2 in a study of renal injury in sepsis (21). Notably, the first two ferroptosis-inducing agents (RSL-3 and erastin) identified in ferroptosis studies initiate the ferroptotic cascade via inhibition of glutathione peroxidase 4 (GPX4) and the cystine/glutamate transporter system (xC-/xCT), respectively, both of which are downstream targets of NRF2 (23). PA is assumed to regulate its downstream proteins, GPX4 and $\mathrm{xCT}$ by activating NRF2, and to interfere with ferroptosis in AKI. The present study aimed to investigate the effect of PA on ferroptosis in mice with ischemia-reperfusion kidney injury, and determine its underlying molecular mechanism of action to provide novel ideas for the treatment of clinical AKI.

\section{Materials and methods}

Reagents and animals. PA (white crystalline powder with purity $\geq 99.9 \%$ ) was purchased from MedChemExpress (cat. no. HY-N0371) and stored away from light at $4^{\circ} \mathrm{C}$. A total of $30 \mathrm{C} 57 \mathrm{BL} / 6$ male mice (8-10 weeks; 20-25 g body weight) were purchased from Chongqing Medical University (Chongqing, China). The housing conditions were as follows: $12 \mathrm{~h}$ light $/ 12 \mathrm{~h}$ dark, $\sim 60 \%$ humidity, $25^{\circ} \mathrm{C}$, free access to drinking water and food and bedding replacement every 3 days; the drinking water and bedding materials were disinfected before use. All animal experiments were performed according to the Guide for the Care and Use of Laboratory Animals, issued by the National Institutes of Health in 1996 (24). The present study was approved by the Institutional Animal Care and Use Committee of Chongqing Medical University (Chongqing, China; approval no. 2018-019).

IRI-AKI model. Mice were fed under the same conditions for 1 week to produce the model of renal ischemia reperfusion injury, as previously described (25). The mice were anesthetized with $50-60 \mathrm{mg} / \mathrm{kg}$ of pentobarbital sodium (cat. no. P3761; Sigma-Aldrich; Merck KGaA) by intraperitoneal injection; the skin at the surgical area was wiped with $70 \%$ alcohol. The incision was positioned at the left and right sides of the spine $(0.5 \mathrm{~cm})$, and the incision length was $1-1.5 \mathrm{~cm}$ along the back. The kidneys were subsequently pulled out from the incision to expose the renal pedicle. A microaneurysm clip was used to clamp the pedicle to block the blood flow to the kidney and induce renal ischemia. Complete ischemia was indicated by a change in the color of the kidney from red to dark purple within a few seconds. After $40 \mathrm{~min}$ of ischemia, the microaneurysm clips were released to allow each kidney to start reperfusion, which was indicated by the change of the kidney color to red. The muscle skin was sutured after the kidney color returned to normal. The entire renal ischemia procedure was completed on a thermostatic pad, and the body temperature of the mice was kept constant at $36.5-37^{\circ} \mathrm{C}$. A total of $0.8 \mathrm{ml}$ warm sterile saline was administered intraperitoneally to each mouse. Each animal was placed on a heating pad until it regained full consciousness and was subsequently returned to its cage. The mice were sacrificed via cervical dislocation $24 \mathrm{~h}$ after surgery, and the blood was collected from the orbital sinus. One-third of the renal tissue was used for histopathological analyses, and the remaining two-thirds were quickly stored at $-80^{\circ} \mathrm{C}$.

Animal groups. C57BL/6 mice were divided into five groups ( $n=6 /$ group) according to a random number table, as follows: Sham group (Sham), model group (IRI), low dose group $\left(\mathrm{IRI}+\mathrm{PA}_{\mathrm{L}}\right)$, moderate dose group (IRI+PA $\mathrm{P}_{\mathrm{M}}$ ) and high dose group $\left(\mathrm{IRI}+\mathrm{PA}_{\mathrm{H}}\right)$. Animals in the three experimental treatment groups received intraperitoneal injection with PA at dosages of 5,10 and $20 \mathrm{mg} / \mathrm{kg}$.bw for 3 days, before the model was induced. The sham and IRI groups were injected with the same volume dimethyl sulfoxide for 3 days before model induction. The modeling method was performed as previously described (25). The bilateral renal pedicels of the mice in the sham group were exposed and treated as aforementioned, but were not clamped. The surgical incision was sutured after $40 \mathrm{~min}$.

Serum biochemical analysis. The mice were sacrificed $24 \mathrm{~h}$ post-surgery, and 0.8-1 ml blood was collected from the orbital sinus, allowed to rest for $30 \mathrm{~min}$ and centrifuged at $900 \mathrm{x} \mathrm{g}$ for $10 \mathrm{~min}$ at $4^{\circ} \mathrm{C}$. The upper serum layer was collected, packed and stored at $-80^{\circ} \mathrm{C}$. Serum creatinine (Scr; cat. no. c011-2-1) and blood urea nitrogen (BUN; cat. no. c013-2-1) were assessed using reagent kits, according to the manufacturer's instructions (Nanjing Jiancheng Bioengineering Institute).

Histopathological examination. At $24 \mathrm{~h}$ post-IRI, portions of the kidney tissues were fixed in $10 \%$ neutral-buffered formalin for 24-48 $\mathrm{h}$ at room temperature, processed routinely by embedding in paraffin. The paraffin-fixed tissue specimens were sliced into $4 \mu \mathrm{m}$ thick sections, which were mounted on glass slides and stained with hematoxylin for $10 \mathrm{~min}$ at room temperature and eosin for $3 \mathrm{~min}$ at room temperature. A BX51 light microscope (Olympus Corporation) was used to detect the renal histopathological changes (magnification, x200 and $\mathrm{x} 400$ ). The Paller score was used to grade renal tubular necrosis in renal IRI (26). A total of 10 non-overlapping areas 
were randomly selected under high magnification using an optical light microscope (magnification, x400), and 10 renal tubules were randomly selected in each field of vision. The renal pathology sections of three mice in each experimental group were randomly selected for observation. A total of 10 visual fields were randomly selected for each pathological section, and 10 renal tubules were scored in each visual field. A total of 100 tubules was observed for each mouse, giving a total of 300 renal tubules observed in each group. The higher the score, the more serious the degree of damage of renal tubules.

Transmission electron microscopy (TEM). Renal tissue was collected $24 \mathrm{~h}$ post-surgery. Following anesthesia, $1 \mathrm{~mm}^{3}$ renal tissue was removed in vivo and soaked in tissue fixation solution composed of $2.5 \%$ glutaraldehyde and phosphoric acid buffer solution (cat. no. G7776; Sigma-Aldrich; Merck KGaA) for $2 \mathrm{~h}$ at $4^{\circ} \mathrm{C}$, dehydrated in gradual ethanol (50-100\%) and propylene oxide, embedded in Epon 812 (cat. no. GP18010; Beijing Zhongjingkeyi Technology Co., Ltd.) for $1 \mathrm{~h}$ at $35^{\circ} \mathrm{C}$ and solidified for $24 \mathrm{~h}$ at $60^{\circ} \mathrm{C}$. Ultrathin sections (thickness, $50-70 \mathrm{~nm}$ ) were placed onto 200-mesh copper grids and stained with $2 \%$ uranyl acetate for $30 \mathrm{~min}$ at room temperature and $0.04 \%$ lead citrate for $15 \mathrm{~min}$ at room temperature. The samples were viewed under an electron microscope (JEM-1400 plus, Japan Electron Optics Laboratory Co., Ltd.; magnification, $\mathrm{x} 5,000$ and $\mathrm{x} 10,000)$. Images were obtained using a SlowScan charge-coupled device camera and iTEM analySIS5.0 software (Olympus Soft Imaging Solutions $\mathrm{GmbH})$.

Antioxidant glutathione (GSH) and lipid peroxidation malondialdehyde (MDA) detection. Kidney tissue (50-100 mg) was collected, and physiological saline solution (cat. no. B020; Nanjing Jiancheng Bioengineering Institute) was added to the tissue at a 1:9 weight $(\mathrm{g})$ :volume $(\mathrm{ml})$ ratio. The tissue was homogenized, to produce $10 \%$ tissue homogenate, which was centrifuged at $13,800 \mathrm{x} \mathrm{g}$ for $15 \mathrm{~min}$ at $4^{\circ} \mathrm{C}$. The supernatant was removed and the total protein content of the supernatant was determined using the bicinchoninic acid method (21). GSH (cat. no. A006-2-1) and MDA (cat. no. A003-2-2) were assessed using the reagent kits, according to the manufacturer's instructions (Nanjing Jiancheng Bioengineering Institute).

Western blotting. Total protein was extracted from mouse kidney tissues using RIPA cell lysis buffer (cat. no. W062-1-1; Nanjing Jiancheng Bioengineering Institute) and the protein concentrations were determined via BCA assay (cat. no. A045-4-2; Nanjing Jiancheng Bioengineering Institute). Equal amounts of proteins $(50 \mu \mathrm{g})$ were separated via SDS-PAGE (Cox-2, 10.0; NRF2, 10.0; xCT, 12.5; HO-1, 12.5; GPx4, 15.0\%) and transferred onto PVDF membranes (GE Healthcare Life Sciences). The membranes were subsequently blocked with $5 \%$ skimmed milk powder for $1 \mathrm{~h}$ at room temperature, and incubated with primary antibodies against the following: Cyclooxygenase 2 (COX-2; 1:1,000; cat. no. ab179800; Abcam), GPX4 (1:5,000; cat. no. ab125066; Abcam), xCT (1:2,000; cat. no. ab175186; Abcam), HO-1 (1:1,000 cat. no. 101147; Gentex) and NRF2 $\left(1: 1,000\right.$; cat. no. D1Z9C; CST) overnight at $4^{\circ} \mathrm{C}$. Membranes were subsequently washed with TBST $(0.1 \%$ Tween-20) three times. Following the primary incubation, membranes were incubated with horseradish peroxidase-conjugated secondary antibodies $(1: 10,000 ; 40295 \mathrm{G}$; BIOSS) for $1 \mathrm{~h}$ at room temperature. The membranes were re-washed three times with TBST and visualized with BeyoECLPlus (cat. no. P00185; Beyotime Institute of Biotechnology), using a Chemi Doc Imaging System (Bio-Rad Laboratories, Inc.) The intensity of protein bands was determined using ImageJ v1.8.0 software (National Institutes of Health). All experiments were performed in triplicate.

Reverse transcription quantitative $(R T-q) P C R$. Total RNA was extracted from the kidneys of mice in different groups using TRIzol ${ }^{\circledR}$ reagent (Takara Bio, Inc.) and cDNA was synthetized using the PrimeScript RT Reagent kit with gDNA Eraser (cat. no. RR047A; Takara Biotechnology Co., Ltd.). Relative levels of the target genes were determined via qPCR using Ultra SYBR Mixture (Takara Biotechnology Co., Ltd.). RT-qPCR was performed in a $25 \mu$ l volume with $2 \mu \mathrm{l}$ cDNA, $400 \mathrm{nM}$ each sense and antisense primer and 12.5 $\mu \mathrm{l}$ Brilliant SYBR Green QPCR Master Mix (Takara Bio, Inc.) on an ABI PRISM 7000 system (Applied Biosystems; Thermo Fisher Scientific, Inc.). The reaction was performed for 40 cycles of denaturation at $95^{\circ} \mathrm{C}$ for $30 \mathrm{sec}$, annealing at $53^{\circ} \mathrm{C}$ for $30 \mathrm{sec}$ and extension at $72^{\circ} \mathrm{C}$ for $10 \mathrm{sec}$. The following primer sequences were used for qPCR: GPX4 forward, 5'-GCCTGGATAAGTACA GGGGTT-3' and reverse, 5'-CATGCAGATCGACTAGCT GAG-3'; NRF2 forward, 5'-TCTTGGAGTAAGTCGAGA AGTGT-3' and reverse, 5'-GTTGAAACTGAGCGAAAA AGGC-3'; xCT forward, 5'-GGCACCGTCATCGGATCA G-3' and reverse, 5'-CTCCACAGGCAGACCAGAAAA-3'; COX-2 forward, 5'-TGAGCAACTATTCCAAACCAGC-3' and reverse, 5'-GCACGTAGTCTTCGATCACTATC-3'; HO-1 forward, 5'-AAGCCGAGAATGCTGAGTTCA-3' and reverse, 5'-GCCGTGTAGATATGGTACAAGGA-3'; and $\beta$-actin forward, 5'-GGCTGTATTCCCCTCCATCG-3' and reverse, 5'-CCAGTTGGTAACAATGCCATGT-3'.

An average value of gene expression after $\beta$-actin normalization was used as a calibrator to determine the relative levels of the target genes. Relative expression levels of the target genes were calculated using the $2^{-\Delta \Delta \mathrm{Cq}}$ method (27).

Statistical analysis. Statistical analysis was performed using GraphPad Prism 7.0 (GraphPad Software, Inc.) and SPSS 23 software (IBM Corp.). Data are presented as the mean \pm standard deviation of three independent repeats, unless stated otherwise. One-way ANOVA followed by Tukey's post hoc test were used to compare differences between multiple groups. $\mathrm{P}<0.05$ was considered to indicate a statistically significant difference.

\section{Results}

PA decreases renal injury in mice with IRI. Scr and BUN are two important indicators of renal function (5). The levels of Scr and BUN were significantly higher in the IRI group compared with those in the sham group $(\mathrm{P}<0.05$; Fig. 1A and B). Treatment with mid-dose and high-dose PA 
A
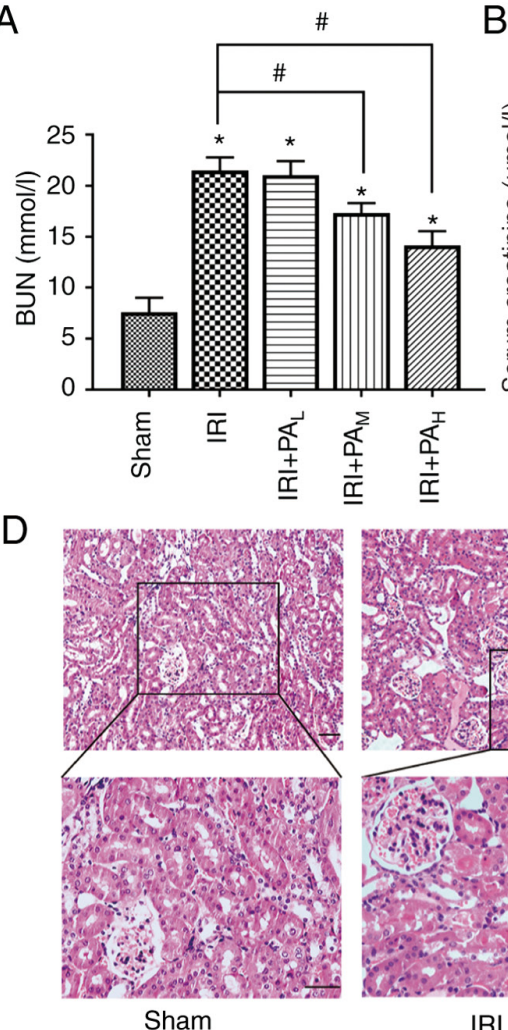

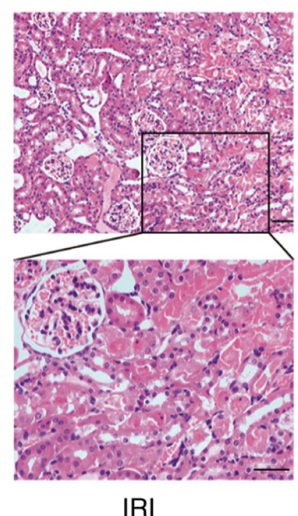

B

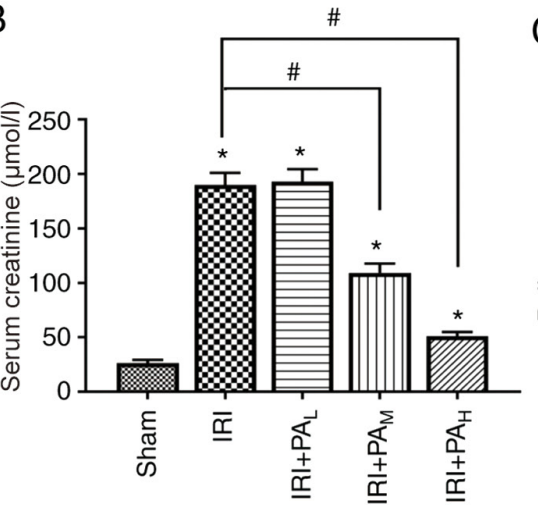

C

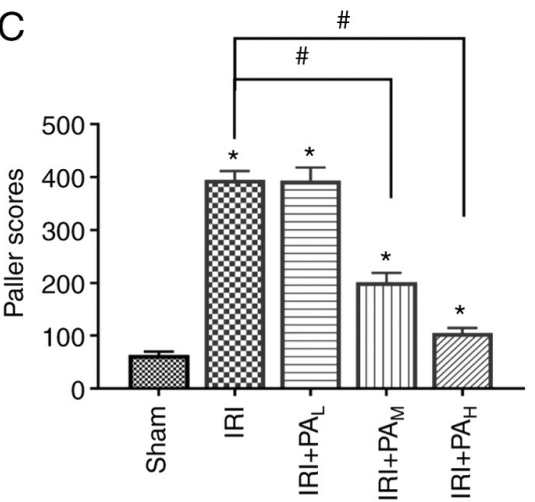

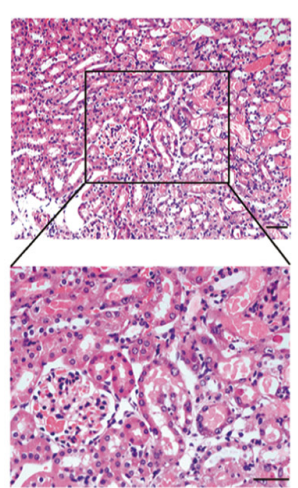

$\mathrm{IRI}+\mathrm{PA}$

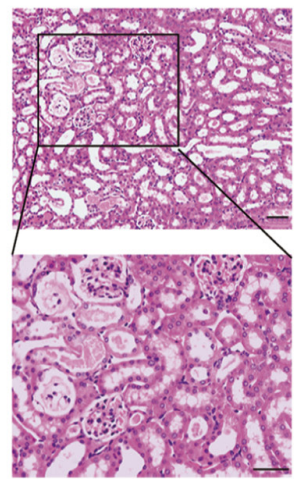

$\mid \mathrm{RI}+\mathrm{PA} \mathrm{A}_{\mathrm{M}}$

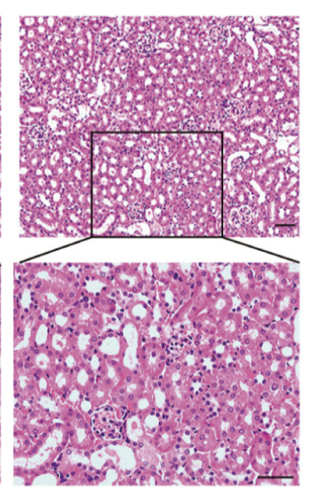

$\mid \mathrm{RI}+\mathrm{PA}_{\mathrm{H}}$

Figure 1. PA alleviates renal injury following ischemia reperfusion. (A) BUN and (B) serum creatinine were measured in mice in the Sham, IRI, IRI+PA, IRI+PA ${ }_{\mathrm{M}}$ and IRI+PA $\mathrm{H}_{\mathrm{H}}$ groups (40 min renal ischemia followed by $24 \mathrm{~h}$ of reperfusion; $\mathrm{n}=6 / \mathrm{group}$ ). (C) Paller scores were used to grade renal tubular injury in

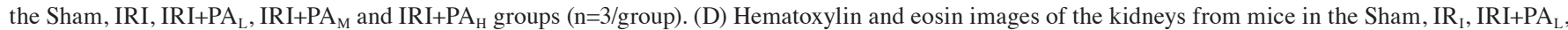
IRI $+\mathrm{PA}_{\mathrm{M}}$ and IRI+PA ${ }_{\mathrm{H}}$ group. Scale bar, $50 \mu \mathrm{m}$. Data are presented as the mean \pm standard deviation. ${ }^{*} \mathrm{P}<0.05$ vs. sham group; ${ }^{\prime} \mathrm{P}<0.05$ vs. IRI group. PA, pachymic acid; BUN, blood urea nitrogen; IRI, ischemia reperfusion injury; ${ }_{\mathrm{L}}$, low dose group; ${ }_{\mathrm{M}}$, moderate dose group; ${ }_{\mathrm{H}}$, high dose group.

significantly mitigated the increases in serum creatinine and urea compared with the IRI group $(\mathrm{P}<0.05)$; however, the levels of serum creatinine and urea did not significantly differ between the light-dose PA treatment group and the IRI group ( $\mathrm{P}>0.05$; Fig. 1A and $\mathrm{B})$.

As presented in Fig. 1D, H\&E staining of kidney tissues in the sham group demonstrated no significant changes in renal tissue structure. Conversely, the IRI group demonstrated edema and loss of renal tubular epithelial cells, tubular dilation, interstitial inflammation, inflammatory cell infiltration, collagen deposition and deposition tube necrosis material (Fig. 1D). The Paller score, an indicator of renal tissue injury (26), was significantly higher in the IRI groups compared with the sham group $(\mathrm{P}<0.05$; Fig. 1C). In particular, moderate-dose and high-dose PA therapy significantly ameliorated these lesions, and the Paller score was significantly lower compared with that in the IRI group $(\mathrm{P}<0.05)$; however, there was no significant difference between the IRI model group and the low-dose group ( $\mathrm{P}>0.05$; Fig. $1 \mathrm{C})$.

Effects of PA on the morphological changes in mitochondria in the renal tissue of mice following renal IRI. TEM demonstrated no significant changes in the mitochondrial structure of the renal tissue in the sham group. In the model group, ferroptosis-associated mitochondrial changes, such as decreased mitochondrial volume, increased membrane density, and decreased or absent mitochondrial cristae, were observed in renal tissue (Fig. 2A). Compared with those in the model group, the mitochondria of the moderate-dose group mainly exhibited edema, and the specific changes characteristic of ferroptosis were rare (Fig. 2A). However, in the high-dose PA group, no characteristic changes of ferroptosis were observed in the mitochondria of the renal tissue, and only mild edema exhibited (Fig. 2A). The changes in the mitochondria in the low-dose group were the same as those in the model group (Fig. 2A).

Effects of PA on GSH and MDA expression in renal IRI. GSH is a necessary cofactor for GPX4 function. GSH synthesis directly affects GPX4 activity. MDA is a product of lipid oxidation, which reflects the degree of intracellular lipid peroxidation and indirectly reflects the occurrence of ferroptosis $(28,29)$. As presented in Fig. 2B, the kidney tissues had significantly lower levels of GSH in the IRI group compared with the sham group. Among the drug treatment groups, GSH expression was significantly higher in the IRI+PA ${ }_{M}$ and IRI+PA ${ }_{H}$ groups compared with the IRI model group $(\mathrm{P}<0.05)$. However, GSH expression did not significantly differ between the IRI $+\mathrm{PA}_{\mathrm{L}}$ and IRI groups $(\mathrm{P}>0.05)$. Conversely, as presented in Fig. 2C, the tissue lipid peroxidation was greater (based on MDA levels) in the IRI group compared with the sham group. Among the drug treatment groups, MDA levels were significantly lower in the IRI+PA and IRI+PA ${ }_{H}$ groups compared with the IRI group $(\mathrm{P}<0.05)$. 


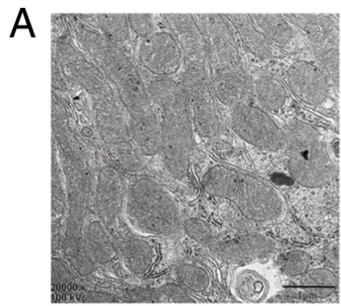

Sham

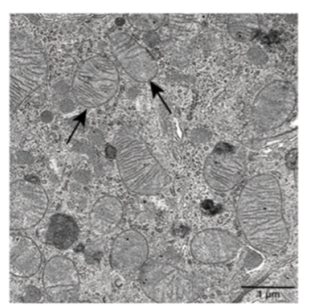

IRI

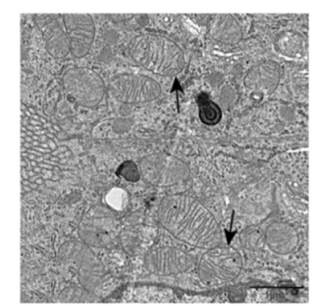

$|R|+P A_{L}$

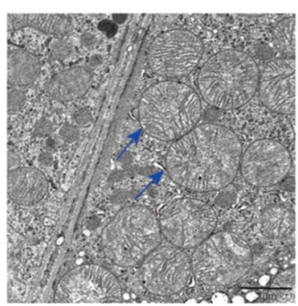

$\mid \mathrm{RI}+\mathrm{PA}_{\mathrm{M}}$

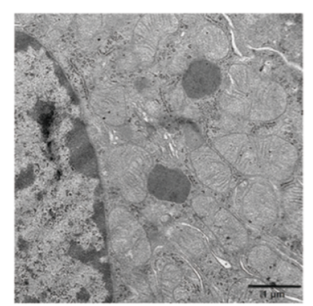

$\mid \mathrm{RI}+\mathrm{PA} \mathrm{H}_{\mathrm{H}}$
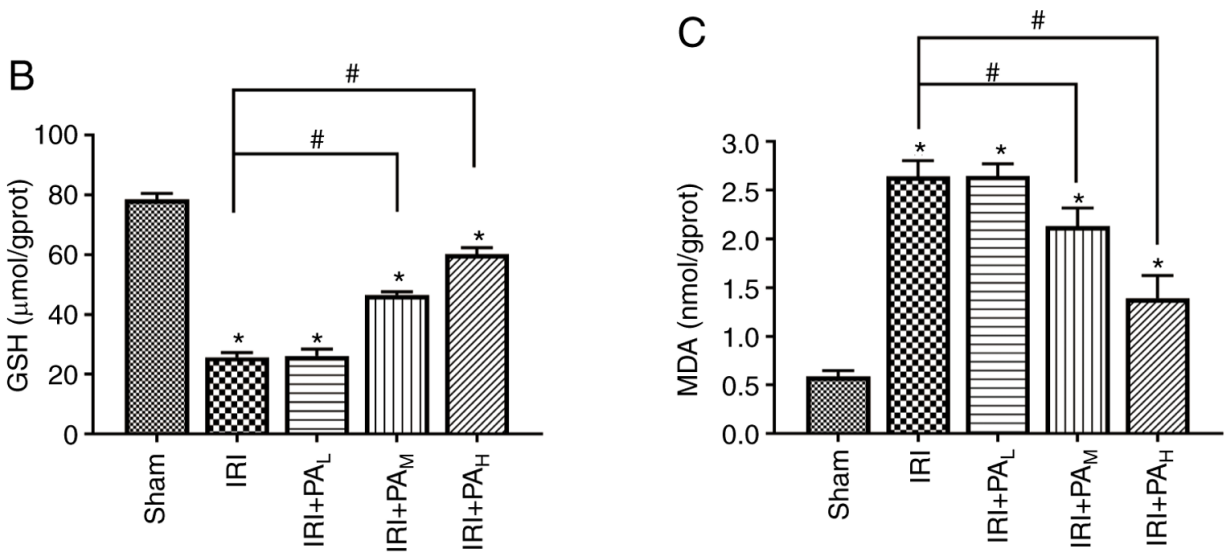

Figure 2. PA decreases ferroptosis in IRI-acute kidney injury. (A) Transmission electron microscopy images in the Sham, IRI, IRI+PA , IRI+PA $_{M}$ and IRI+PA groups (40 min renal ischemia followed by $24 \mathrm{~h}$ of reperfusion). Magnification, x20,000; scale bar, $1 \mu \mathrm{m}$. The black arrows indicate that the mitochondrial volume decreased, membrane density increased and decreased, or disappeared mitochondrial cristae were observed in renal tissue. The blue arrows indicate mitochondrial edema. The levels of (B) GSH and (C) MDA in renal tissues were assessed (n=6/group). Data are presented as the mean \pm standard deviation. ${ }^{*} \mathrm{P}<0.05$ vs. sham group; ${ }^{\#} \mathrm{P}<0.05$ vs. IRI group. PA, pachymic acid; IRI, ischemia reperfusion injury; ${ }_{\mathrm{L}}$, low dose group; ${ }_{\mathrm{M}}$, moderate dose group; ${ }_{\mathrm{H}}$, high dose group; GSH, glutathione; MDA, malondialdehyde.

Notably, no significant differences were observed in the levels of MDA between the IRI+PA $\mathrm{L}_{\mathrm{L}}$ and IRI groups $(\mathrm{P}>0.05)$.

$P A$ regulates the expression of ferroptosis-related proteins in renal IRI. In the present study, the expression levels of the ferroptosis-related proteins GPX4, xCT and HO-1 were detected via western blotting (Fig. 3A and B) and RT-qPCR (Fig. 3C) analyses. The results demonstrated that the protein levels of GPX4, xCT and HO-1 were significantly downregulated in the IRI group compared with the sham group $(\mathrm{P}<0.05)$ (Fig. 3A and B). Similar results were demonstrated following RT-qPCR analysis. In addition, treatment with moderate-dose and high-dose PA significantly increased the protein and mRNA expression levels of GPX4, xCT and HO-1 compared with the IRI group $(\mathrm{P}<0.05)$. However, no significant differences in protein and mRNA expression levels of GPX4, xCT and HO-1 were observed between the IRI and low-dose PA treatment groups $(\mathrm{P}>0.05$; Fig. $3 \mathrm{~B}$ and $\mathrm{C})$.

The results demonstrated that protein and mRNA levels of the ferroptosis-associated lipid peroxidation protein, COX-2 were significantly upregulated during IRI (Fig. 4). Treatment with moderate-dose and high-dose PA significantly decreased COX-2 expression compared with the IRI group $(\mathrm{P}<0.05)$, and no significant differences were observed between the IRI and low-dose PA treatment groups ( $\mathrm{P}>0.05$; Fig. 4).

$P A$ promotes activation of the NRF2 signaling pathway in renal IRI. To investigate the effect of PA on the NRF2 signaling pathway, the protein and mRNA levels of NRF2 were assessed (Fig. 5). The results demonstrated that NRF2 expression was significantly lower in the IRI group compared with the sham group. In addition, NRF2 expression was significantly higher in the IRI+PA ${ }_{M}$ and IRI+PA ${ }_{H}$ groups compared with the IRI group $(\mathrm{P}<0.05)$; however, no significant differences were observed between the IRI+PA ${ }_{L}$ and IRI groups $(\mathrm{P}>0.05)$. Furthermore, treatment with PA also increased NRF2 expression in a dose-dependent manner (Fig. 5).

\section{Discussion}

Previous studies have demonstrated that PA ameliorates renal injury in AKI and chronic kidney disease via anti-inflammatory and antioxidative stress $(21,22)$. However, whether PA inhibits ferroptosis has not yet been investigated. To the best of our knowledge, the present study was the first to investigate the effect of PA on ferroptosis in IRI-AKI. In the present study, bilateral renal pedicle clamping in mice for $40 \mathrm{~min}$ followed by reperfusion for $24 \mathrm{~h}$ was used to successfully establish IRI and induce AKI. The results demonstrated that Scr and BUN levels were significantly elevated in the model group, and renal tubules were significantly damaged, based on H\&E staining and the Paller score. Thus, the IRI-AKI model was constructed. Notably, administration of PA significantly decreased the IRI enhanced Scr and BUN serum levels, and alleviated kidney injury in a dose-dependent manner.

GPX4 serves an important role in ferroptosis (30). Small molecules, such as erastin, RSL-3 and RSL-5 (13), through different signaling pathways, directly or indirectly inhibit GPX4 activity, thus decreasing cell antioxidant capacity, 
A
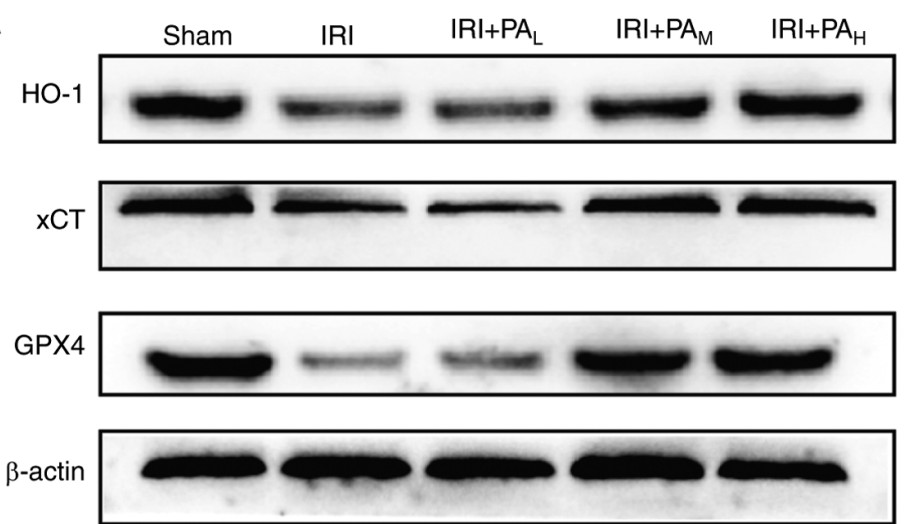

B
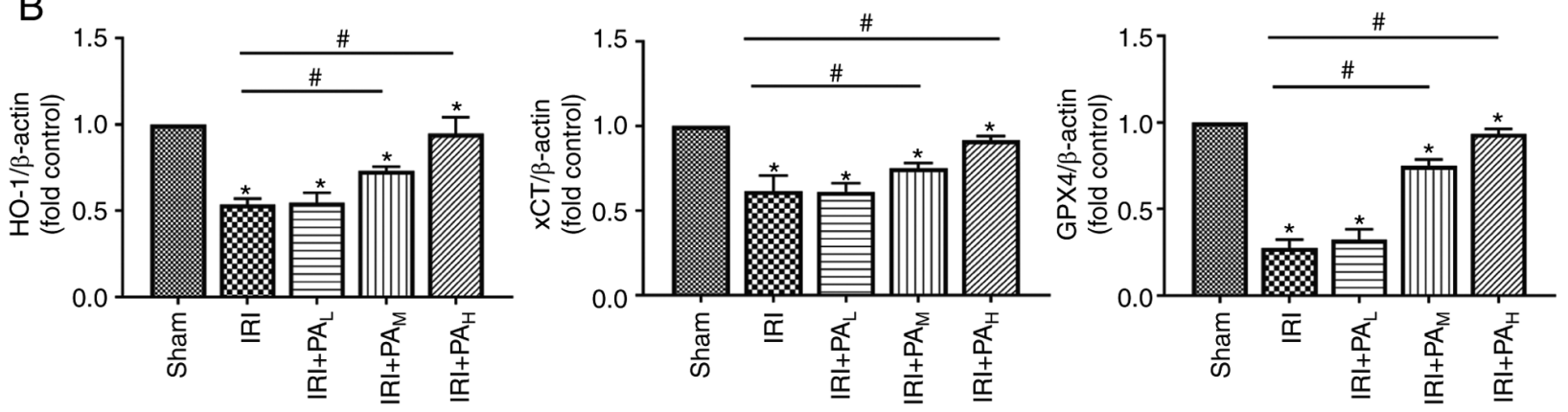

C
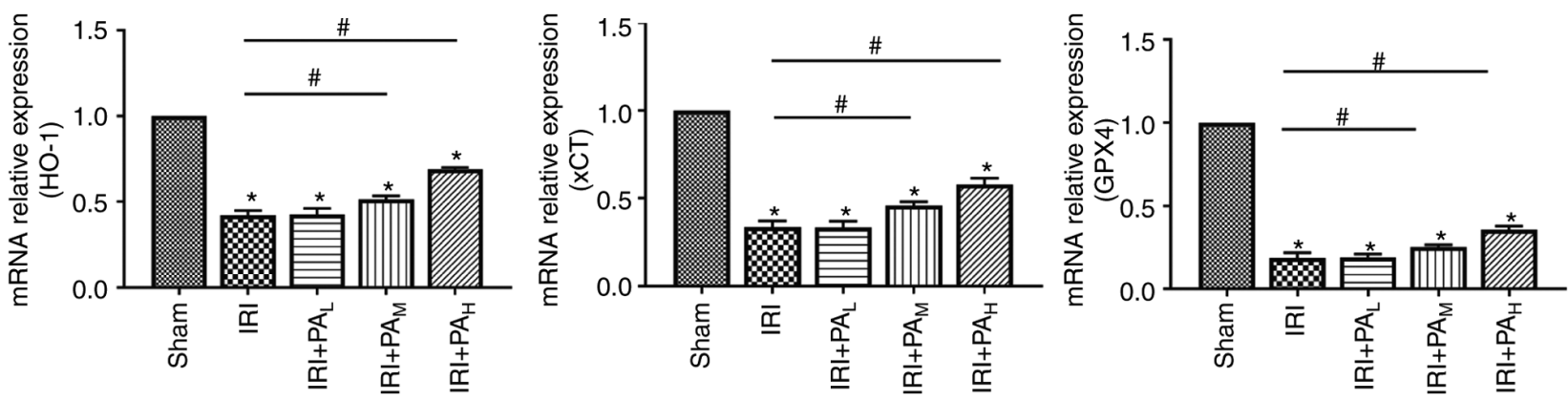

Figure 3. PA increases the expression levels the ferroptosis related proteins, GPX4, xCT and HO-1. (A) Western blotting was performed to detect the expression levels of the ferroptosis related proteins, GPX4, xCT and HO-1 in IRI-acute kidney injury mice (n=3). (B) Relative expression of gray values for GPX4, xCT and HO-1. (C) Reverse transcription-quantitative PCR analysis was performed to detect the mRNA expression levels of the ferroptosis related proteins ( $\mathrm{n}=3$ ). Data are presented as the mean \pm standard deviation. ${ }^{*} \mathrm{P}<0.05$ vs. sham group; ${ }^{\sharp} \mathrm{P}<0.05$ vs. IRI group. PA, pachymic acid; GPX4, glutathione peroxidase 4; $\mathrm{xCT}$, glutamate transporter system; HO-1, heme oxygenase 1 ; IRI, ischemia reperfusion injury; ${ }_{\mathrm{L}}$, low dose group; ${ }_{\mathrm{M}}$, moderate dose group; ${ }_{\mathrm{H}}$, high dose group.

causing reactive oxygen species overload and membrane lipid peroxidation reactions, and ultimately damaging cell membrane integrity and causing cell ferroptosis (13). In GPX4 knockout mice, GPX4 deficiency leads to spontaneous acute renal failure and an increased rate of early mortality, whereas ferroptosis of renal tubular epithelial cells is the main cause of renal failure in GPX4 knockout mice (30). Thus, increasing GPX4 activity effectively inhibits ferroptosis. The synthesis of GSH, a cofactor necessary for GPX4 function, directly increases GPX4 activity. GSH synthesis is influenced by cystine. The transport of cystine from the extracellular to the intracellular space is largely dependent on the cystine/glutamate reverse transporter (system xc-) on the cell membrane. This transporter is a heterodimer composed of two subunits, SLC7A11 (xCT) and SLC3A2. It mainly transports glutamate to the extracellular space and mediates cystine entry into the cell (28). Inhibiting the activity of system xc- affects the synthesis of GSH by inhibiting the absorption of cystine, thereby leading to inactivity of GPX4, a decrease in cell antioxidant capacity, accumulation of lipid reactive oxygen species, and ultimately oxidative damage and ferroptosis (31). Yang et al (32) confirmed that prostaglandin-endoperoxide synthase 2 (PTGS2), encoding COX-2, is a suitable marker for the lipid peroxidation that occurs during GPX4-regulated ferroptosis. Thus, PTGS2 upregulation has been suggested to be a downstream marker of ferroptosis. Enhancing system xc-, promoting the absorption of cystine, increasing the production of GSH and increasing the activity of GPX4 may inhibit ferroptosis and decrease renal injury $(32,33)$. The results of the present study demonstrated that the system xc- activity decreased, GSH synthesis decreased, GPX4 activity decreased, MDA and Cox-2 expression increased, and ferroptosis occurred in IRI-AKI mice. In addition, treatment with PA significantly 
A
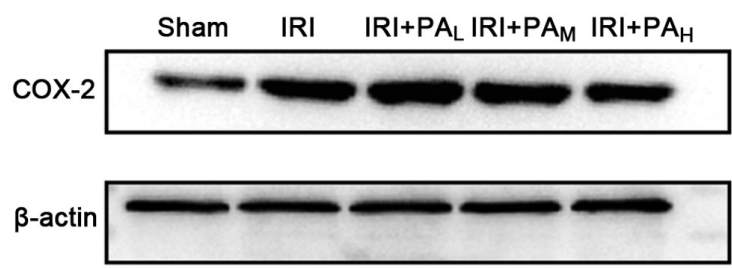

B

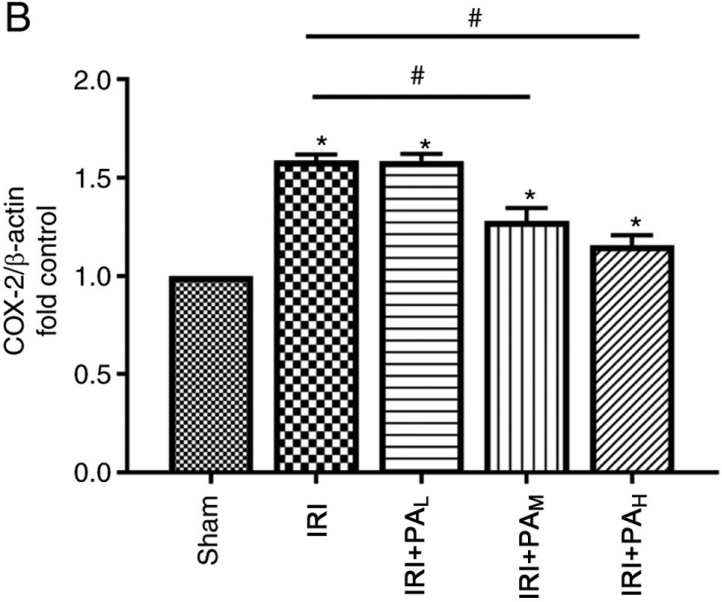

C

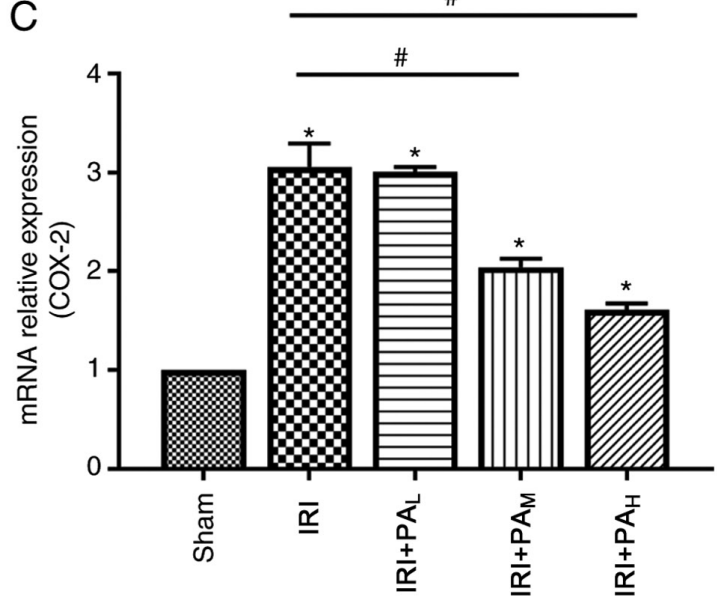

Figure 4. PA decreases the expression levels of the ferroptosis related protein, COX-2. (A) Western blotting was performed to detect the expression levels of the ferroptosis lipid peroxidation marker protein, COX-2 in IRI-acute kidney injury mice. (B) Relative expression of gray values for COX-2. (C) Reverse transcription-quantitative PCR analysis was performed to detect mRNA COX-2 expression $(n=3)$. Data are presented as the mean \pm standard deviation. ${ }^{*} \mathrm{P}<0.05$ vs. sham group; ${ }^{*} \mathrm{P}<0.05$ vs. IRI group. PA, pachymic acid; COX-2, cyclooxygenase 2; IRI, ischemia reperfusion injury; ${ }_{\mathrm{L}}$, low dose group; ${ }_{\mathrm{M}}$, moderate dose group; ${ }_{\mathrm{H}}$, high dose group.

A
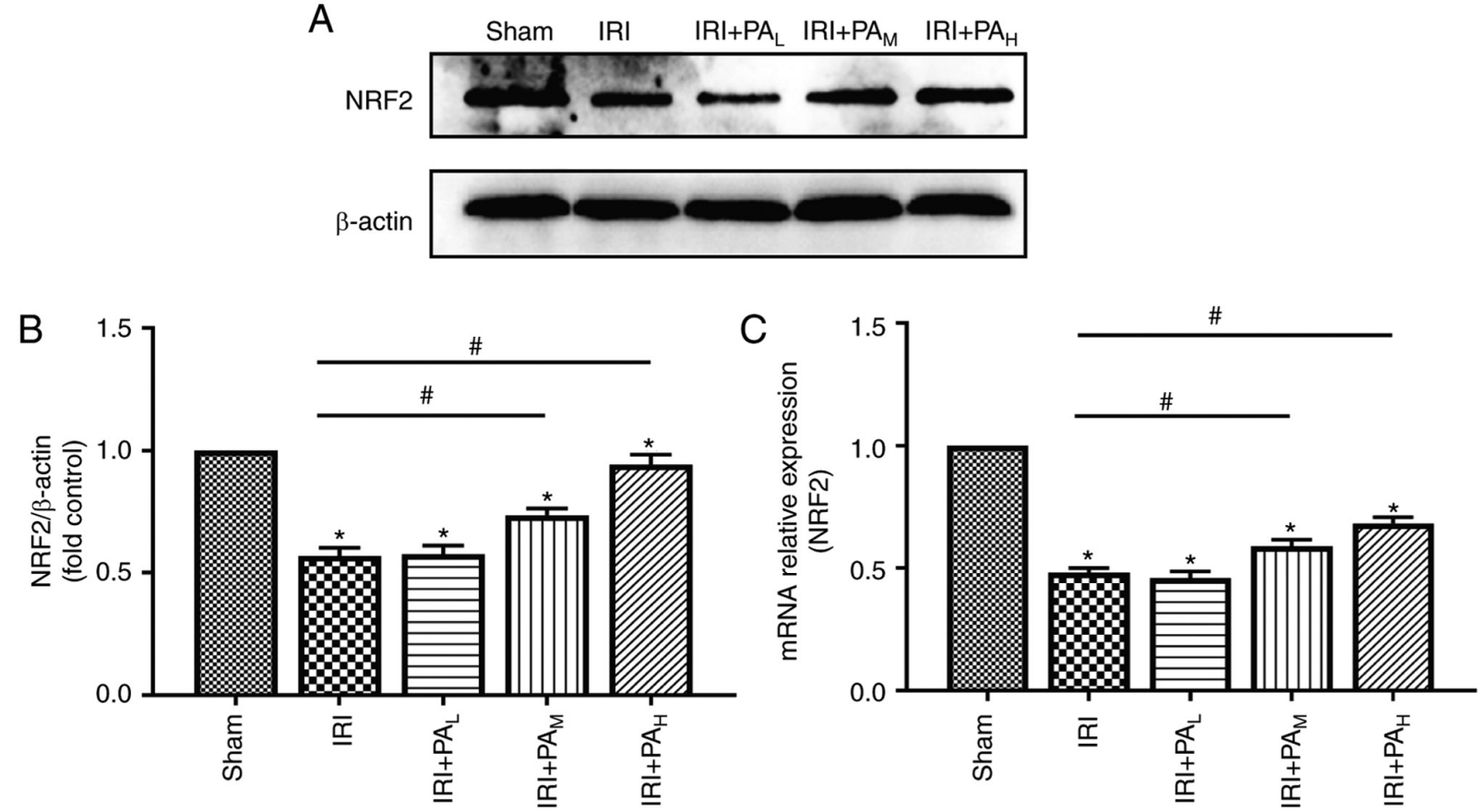

Figure 5. PA activates the NRF2 signaling pathway. (A) Western blotting was performed to detect NRF2 protein expression in IRI-acute kidney injury mice. (B) Relative expression of gray values for NRF2 $(n=3)$. (C) Reverse transcription-quantitative PCR analysis was performed to detect mRNA NRF2 expression $(\mathrm{n}=3)$. Data are presented as the mean \pm standard deviation. ${ }^{*} \mathrm{P}<0.05$ vs. sham group; ${ }^{*} \mathrm{P}<0.05$ vs. IRI group. PA, pachymic acid; NRF2, nuclear factor erythroid derived 2 like 2; IRI, ischemia reperfusion injury; ${ }_{\mathrm{L}}$, low dose group; ${ }_{\mathrm{M}}$, moderate dose group; ${ }_{\mathrm{H}}$, high dose group.

increased the expression levels of GSH, $\mathrm{xCT}$ and GPX4, and decreased the expression levels of MDA and Cox-2, thus suggesting that treatment with moderate and high doses of
PA enhances system xc- activity, promotes the absorption of cystine, increases GSH production, increases GPX4 activity and inhibits ferroptosis. 


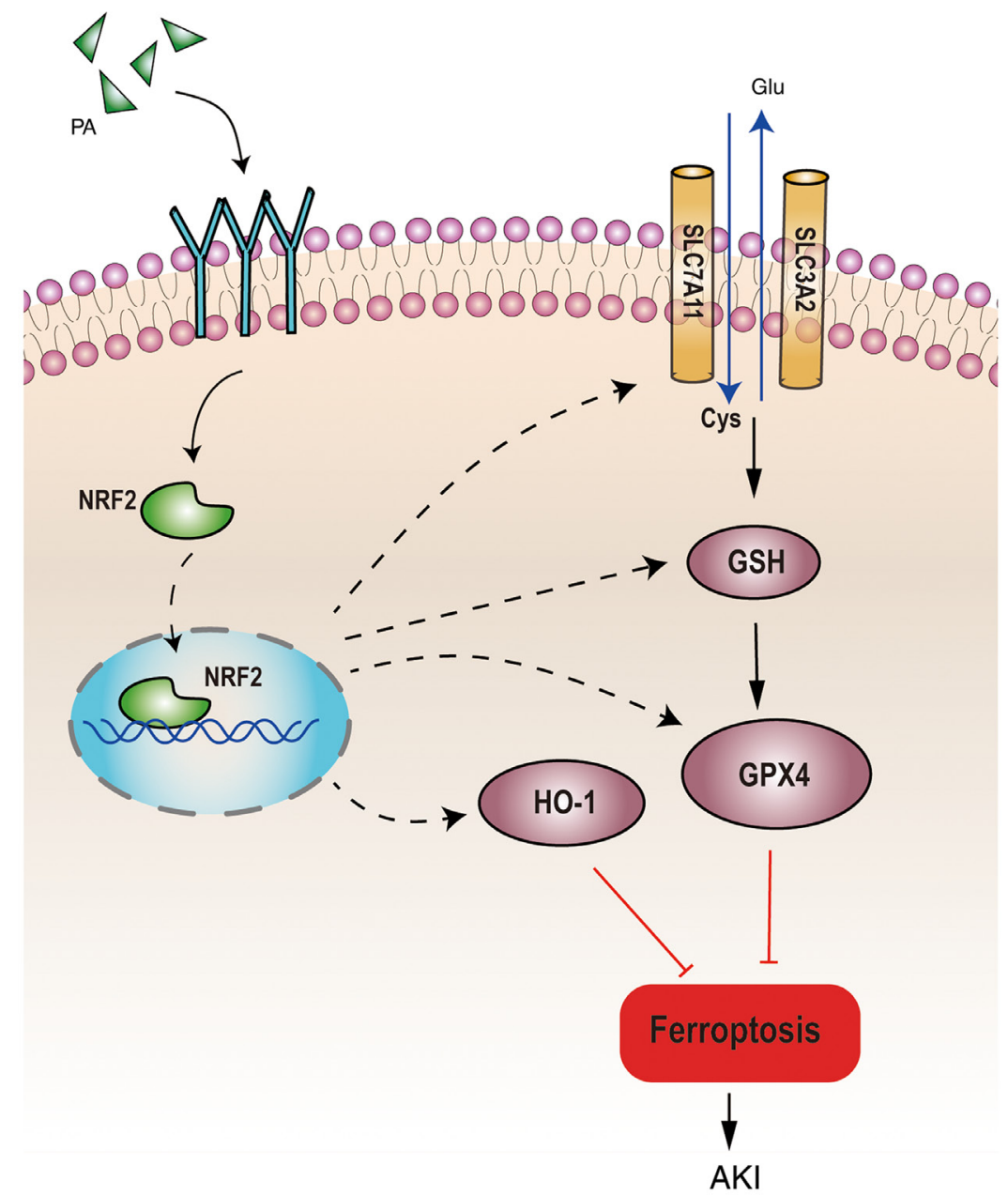

Figure 6. Schematic diagram of proposed molecular mechanisms for PA regulating ferroptosis in IRI-AKI. PA can improve renal function and decrease renal injury in IRI-AKI mice by activating the NRF2 signaling pathway, GSH and downstream ferroptosis-related proteins (GPX4, $\mathrm{xCT}$ and HO-1) to inhibit ferroptosis in the kidney. PA, pachymic acid; IRI, ischemia reperfusion injury; AKI, acute kidney injury; NRF2, nuclear factor erythroid derived 2 like 2; GSH, glutathione; GPX4, glutathione peroxidase 4; HO-1, heme oxygenase 1; xCT, glutamate transporter system; Glu, glutamic acid; Cys, cysteine.

NRF2, a stress-inducible transcription factor, has emerged as a key regulator of both lipid peroxidation and ferroptosis (23). Notably, a number of the proteins and enzymes (such as GPX4, $\mathrm{xCT}$ and the glutamate-cysteine ligase catalytic and modifier subunits) responsible for preventing lipid peroxidation, and thus initiation of ferroptosis, are NRF2 target genes (23). NRF2 serves a key role in regulating iron/heme metabolism (34). Following activation of NRF2, NRF2 upregulates iron metabolism related proteins involved in ferroptosis, such as ferritin (Ferritin light chain and Ferritin heavy polypeptide 1), the intracellular iron storage proteins (35), iron chelatase and HO-1 (36). Adedoyin et al (37) reported that HO-1 in proximal renal tubular epithelial cells can resist ferroptosis induced by Erastin in AKI, thus, decreasing AKI. In addition, NRF2 regulates proteases involved in GSH synthesis and metabolism, such as glutamine-cysteine ligase catalytic and regulatory subunits, glutathione synthase and a subunit of the cystine/glutamate transporter xCT (38-40). GPX4 is a target regulatory gene of NRF2 (41). Li et al (42) demonstrated that pretreatment with Roxadustat (FG-4592) attenuates folic acid-induced kidney injury through anti-ferroptosis via the Akt/GSK-3 $\beta / N R F 2$ pathway. The results of the present study demonstrated that administration of moderate and high doses of PA directly or indirectly promoted the activation of the NRF2 signaling pathway, upregulated the expression levels of the downstream ferroptosis regulated proteins, GPX4, xCT and HO-1, increased GSH levels in renal tissue, and decreased the levels of the ferroptosis-associated lipid peroxidation protein, COX-2 and the lipid oxidation indicator, MDA.

Taken together, the results of the present study suggest that PA may improve renal function and decrease renal injury in IRI-AKI mice. This protective effect may be associated with the inhibition of ferroptosis in the kidneys through direct or indirect activation of NRF2, and upregulation of the downstream ferroptosis-related proteins, GPX4, xCT and HO-1 (Fig. 6). However, the present study is not without limitations. The effect of PA on ferroptosis has not been extensively studied. Thus, prospective studies should use erastin, a selective inhibitor of system xc-, to inhibit the activity of $\mathrm{xCT}$ in mice, and subsequently verify whether inhibition of $\mathrm{xCT}$ induces ferroptosis in mouse kidney cells, thus leading to AKI, and whether PA inhibits ferroptosis mainly through the xCT-GPX4 pathway. Further mechanistic studies must be performed in vitro. In addition, PA should be used to treat renal tubular epithelial cells to verify the effect of PA on ferroptosis in vitro. 


\section{Acknowledgements}

The authors would like to thank Professor Ling Zhang (Department of Nephrology, The Second Affiliated Hospital, Chongqing Medical University) for help designing the study and guiding the animal model construction.

\section{Funding}

The present study was supported by grants from the National Natural Science Foundation of China (grant nos. 81873604 and 81000299), the Venture and Innovation Support Program for Chongqing Overseas Returnees (grant no. cx2018038), the Fourth Batch of Chongqing Young and Middle-aged Medical TopTalent Fund [grant no. (2018)230] and the Kuanren Talents Program of The Second Affiliated Hospital of Chongqing Medical University (grant no. KY2019G008).

\section{Availability of data and materials}

The datasets used and/or analyzed during the current study are available from the corresponding author on reasonable request.

\section{Authors' contributions}

GPJ and YJL designed the present study, performed most of the experiments, and drafted the initial manuscript. XJZ and LLH analyzed the data. XHL helped analyze and interpret the data, and revised and corrected the manuscript for important intellectual content. All authors read and approved the final manuscript.

\section{Ethics approval and consent to participate}

All animal experiments were performed according to the Guide for the Care and Use of Laboratory Animals, issued by the National Institutes of Health in 1996.The present study was approved by the Institutional Animal Care and Use Committee of Chongqing Medical University (Chongqing, China; approval no. 2018-019).

\section{Patient consent for publication}

Not applicable.

\section{Competing interests}

The authors declare that they have no competing interests.

\section{References}

1. Hoste EAJ, Kellum JA, Selby NM, Zarbock A, Palevsky PM, Bagshaw SM, Goldstein SL, Cerdá J and Chawla LS: Global epidemiology and outcomes of acute kidney injury. Nat Rev Nephrol 14: 607-625, 2018.

2. Kaddourah A, Basu RK, Bagshaw SM and Goldstein SL; AWARE Investigators: Epidemiology of acute kidney injury in critically Ill children and young adults. N Engl J Med 376: 11-20, 2017.

3. Chertow GM, Burdick E, Honour M, Bonventre JV and Bates DW: Acute kidney injury, mortality, length of stay, and costs in hospitalized patients. J Am Soc Nephrol 16: 3365-3370, 2005.
4. Susantitaphong P, Cruz DN, Cerda J, Abulfaraj M, Alqahtani F, Koulouridis I and Jaber BL; Acute Kidney Injury Advisory Group of the American Society of Nephrology: World incidence of AKI: A meta-analysis. Clin J Am Soc Nephrol 8: 1482-1493, 2013.

5. Ronco C, Bellomo R and Kellum JA: Acute kidney injury. Lancet 394: 1949-1964, 2019.

6. James MT, Bhatt M, Pannu N and Tonelli M: Long-term outcomes of acute kidney injury and strategies for improved care. Nat Rev Nephrol 16: 193-205, 2020.

7. Han SJ and Lee HT: Mechanisms and therapeutic targets of ischemic acute kidney injury. Kidney Res Clin Pract 38: 427-440, 2019.

8. Priante G, Gianesello L, Ceol M, Del Prete D and Anglani F: Cell death in the kidney. Int J Mol Sci 20: 3598, 2019.

9. Martin-Sanchez D, Poveda J, Fontecha-Barriuso M, Ruiz-Andres O, Sanchez-Niño MD, Ruiz-Ortega M, Ortiz A and Sanz AB: Targeting of regulated necrosis in kidney disease. Nefrologia 38: 125-135, 2018.

10. Kers J, Leemans JC and Linkermann A: An overview of pathways of regulated necrosis in acute kidney injury. Semin Nephrol 36: 139-152, 2016.

11. Pefanis A, Ierino FL, Murphy JM and Cowan PJ: Regulated necrosis in kidney ischemia-reperfusion injury. Kidney Int 96: 291-301, 2019.

12. Hu Z, Zhang H, Yang SK, Wu X, He D, Cao K and Zhang W: Emerging role of ferroptosis in acute kidney injury. Oxid Med Cell Longev 2019: 8010614, 2019.

13. Dixon SJ, Lemberg KM, Lamprecht MR, Skouta R, Zaitsev EM, Gleason CE, Patel DN, Bauer AJ, Cantley AM, Yang WS, et al: Ferroptosis: An iron-dependent form of nonapoptotic cell death. Cell 149: 1060-1072, 2012.

14. Li J, Cao F, Yin HL, Huang ZJ, Lin ZT, Mao N, Sun B and Wang G: Ferroptosis: Past, present and future. Cell Death Dis 11: $88,2020$.

15. Linkermann A, Skouta R, Himmerkus N, Mulay SR, Dewitz C, De Zen F, Prokai A, Zuchtriegel G, Krombach F, Welz PS, et al: Synchronized renal tubular cell death involves ferroptosis. Proc Natl Acad Sci USA 111: 16836-16841, 2014.

16. Ling H, Zhang Y, Ng KY and Chew EH: Pachymic acid impairs breast cancer cell invasion by suppressing nuclear factor- $\kappa \mathrm{B}$-dependent matrix metalloproteinase-9 expression. Breast Cancer Res Treat 126: 609-620, 2011.

17. Li FF, Yuan Y, Liu Y, Wu QQ, Jiao R, Yang Z, Zhou MQ and Tang QZ: Pachymic acid protects H9c2 cardiomyocytes from lipopolysaccharide-induced inflammation and apoptosis by inhibiting the extracellular signal-regulated kinase $1 / 2$ and p38 pathways. Mol Med Rep 12: 2807-2813, 2015.

18. Li JY, Wu HX and Yang G: Pachymic acid improves survival and attenuates acute lung injury in septic rats induced by cecal ligation and puncture. Eur Rev Med Pharmacol Sci 21: 1904-1910, 2017.

19. Li TH, Hou CC, Chang CL and Yang WC: Anti-hyperglycemic properties of crude extract and triterpenes from poria cocos. Evid Based Complement Alternat Med 2011: 128402, 2011.

20. Shah VK, Choi JJ, Han JY, Lee MK, Hong JT and Oh KW: Pachymic acid enhances pentobarbital-induced sleeping behaviors via GABAA-ergic systems in mice. Biomol Ther (Seoul) 22: 314-320, 2014.

21. Cai ZY, Sheng ZX and Yao H: Pachymic acid ameliorates sepsis-induced acute kidney injury by suppressing inflammation and activating the Nrf2/HO-1 pathway in rats. Eur Rev Med Pharmacol Sci 21: 1924-1931, 2017.

22. Chen DQ, Feng YL, Chen L, Liu JR, Wang M, Vaziri ND and Zhao YY: Poricoic acid A enhances melatonin inhibition of AKI-to-CKD transition by regulating Gas6/AxlNFuB/Nrf2 axis. Free Radic Biol Med 134: 484-497, 2019.

23. Dodson M, Castro-Portuguez R and Zhang DD: NRF2 plays a critical role in mitigating lipid peroxidation and ferroptosis. Redox Biol 23: 101107, 2019.

24. National Research Council (US) Institute for Laboratory Animal Research: Guide for the Care and Use of Laboratory Animals. Washington (DC): National Academies Press (US); 1996. https://www.ncbi.nlm.nih.gov/books/NBK232589/ doi: $10.17226 / 5140$.

25. Wei Q and Dong Z: Mouse model of ischemic acute kidney injury: Technical notes and tricks. Am J Physiol Renal Physiol 303: F1487-F1494, 2012.

26. Paller MS, Hoidal JR and Ferris TF: Oxygen free radicals in ischemic acute renal failure in the rat. J Clin Invest 74: 1156-1164, 1984. 
27. Livak KJ and Schmittgen TD: Analysis of relative gene expression data using real-time quantitative PCR and the 2(-Delta Delta C(T)) method. Methods 25: 402-408, 2001

28. Xie Y, Hou W, Song X, Yu Y, Huang J, Sun X, Kang R and Tang D: Ferroptosis: Process and function. Cell Death Differ 23: 369-379, 2016.

29. Su L, Jiang X, Yang C, Zhang J, Chen B, Li Y, Yao S, Xie Q, Gomez H, Murugan R and Peng Z: Pannexin 1 mediates ferroptosis that contributes to renal ischemia/reperfusion injury. J Biol Chem 294: 19395-19404, 2019.

30. Friedmann Angeli JP, Schneider M, Proneth B, Tyurina YY, Tyurin VA, Hammond VJ, Herbach N, Aichler M, Walch A, Eggenhofer E, et al: Inactivation of the ferroptosis regulator Gpx4 triggers acute renal failure in mice. Nat Cell Biol 16: 1180-1191, 2014.

31. Dixon SJ, Patel DN, Welsch M, Skouta R, Lee ED, Hayano M, Thomas AG, Gleason CE, Tatonetti NP, Slusher BS and Stockwell BR: Pharmacological inhibition of cystine-glutamate exchange induces endoplasmic reticulum stress and ferroptosis. Elife 3: e02523, 2014.

32. Yang WS, SriRamaratnam R, Welsch ME, Shimada K, Skouta R, Viswanathan VS, Cheah JH, Clemons PA, Shamji AF, Clish CB, et al: Regulation of ferroptotic cancer cell death by GPX4. Cell 156: 317-331, 2014.

33. Cao JY and Dixon SJ: Mechanisms of ferroptosis. Cell Mol Life Sci 73: 2195-2209, 2016.

34. Kerins MJ and Ooi A: The roles of NRF2 in modulating cellular iron homeostasis. Antioxid Redox Signal 29: 1756-1773, 2018.

35. Harada N, Kanayama M, Maruyama A, Yoshida A, Tazumi K, Hosoya T, Mimura J, Toki T, Maher JM, Yamamoto M and Itoh K: Nrf2 regulates ferroportin 1-mediated iron efflux and counteracts lipopolysaccharide-induced ferroportin $1 \mathrm{mRNA}$ suppression in macrophages. Arch Biochem Biophys 508: 101-109, 2011.

36. Alam J, Stewart D, Touchard C, Boinapally S, Choi AM and Cook JL: Nrf2, a Cap'n'Collar transcription factor, regulates induction of the heme oxygenase-1 gene. J Biol Chem 274: 26071-26078, 1999.
37. Adedoyin O, Boddu R, Traylor A, Lever JM, Bolisetty S, George JF and Agarwal A: Heme oxygenase-1 mitigates ferroptosis in renal proximal tubule cells. Am J Physiol Renal Physiol 314: F702-F714, 2018.

38. Chan JY and Kwong M: Impaired expression of glutathione synthetic enzyme genes in mice with targeted deletion of the Nrf2 basic-leucine zipper protein. Biochim Biophys Acta 1517: 19-26, 2000

39. Yang H, Magilnick N, Lee C, Kalmaz D, Ou X, Chan JY and Lu SC: Nrf1 and Nrf2 regulate rat glutamate-cysteine ligase catalytic subunit transcription indirectly via NF-kappaB and AP-1. Mol Cell Biol 25: 5933-5946, 2005.

40. Sasaki H, Sato H, Kuriyama-Matsumura K, Sato K, Maebara K, Wang $\mathrm{H}$, Tamba $\mathrm{M}$, Itoh $\mathrm{K}$, Yamamoto $\mathrm{M}$ and Bannai $\mathrm{S}$ : Electrophile response element-mediated induction of the cystine/glutamate exchange transporter gene expression. J Biol Chem 277: 44765-44771, 2002.

41. Salazar M, Rojo AI, Velasco D, de Sagarra RM and Cuadrado A: Glycogen synthase kinase-3beta inhibits the xenobiotic and antioxidant cell response by direct phosphorylation and nuclear exclusion of the transcription factor Nrf2. J Biol Chem 281: 14841-14851, 2006.

42. Li X, Zou Y, Xing J, Fu YY, Wang KY, Wan PZ and Zhai XY: Pretreatment with roxadustat (FG-4592) attenuates folic acid-induced kidney injury through antiferroptosis via Akt/GSK-3 $\beta /$ Nrf2 pathway. Oxid Med Cell Longev 2020: $6286984,2020$.

This work is licensed under a Creative Commons Attribution-NonCommercial-NoDerivatives 4.0 International (CC BY-NC-ND 4.0) License. 